\title{
Block Josephus Problem: When the reality is more cruel than the old story
}

\author{
Jang-Woo Park (iD, Ali Dogan* (D), Ricardo Teixeira (i) \\ School of Arts and Sciences, University of Houston-Victoria, Victoria TX 77901, USA
}

\begin{abstract}
In the Josephus Problem, there are $n$ people numbered from 0 to $n-1$ around a circle and proceeding around the circle every second person is executed until no one survives. Determining where to stand on the circle to be the last survivor is called the Josephus Problem. In this paper, we present a generalized version of the Josephus Problem and study cases where multiple executions occur at each iteration. Especially, we focus on the Block Josephus problem where the number of skips and the number of executions are the same. In particular, we present nonrecursive formulas for the initial positions of survivors in the Block Josephus Problem.
\end{abstract}

Mathematics Subject Classification (2020). 68R01, 97N70

Keywords. Josephus problem, discrete mathematics, function iterations

\section{Introduction}

The Josephus Problem is named after Flavius Josephus who was a famous historian lived in the first century. During the first Jewish-Roman War [4], Josephus and his 40 soldiers trapped in a cave and he persuaded his soldiers to take part in a lethal game of chance and chose suicide over capture. According to the legend his mathematical skills helped him survive in this lethal game of chance.

The statement of the original Josephus Problem is as follows: There are $n$ people around a circle, and proceeding around the circle every second person is executed until no one survives in $n$ iterations. The Josephus Problem is to identify the initial position to be the last survivor in this scenario.

There are many generalizations of this problem. Especially skipping several people and executing one person is the one that has been studied extensively in $[1-3,5,7,9,11,12]$ and [13]. Other versions of the problem include the Feline Josephus Problem in which each person has multiple lives was studied by Ruskey and Williams in [8], and a version in which the number of people skipped varies with each iteration was studied by Sharma et al in [10].

\footnotetext{
*Corresponding Author.

Email addresses: ParkJ1@uhv.edue (J. Park), DoganA@uhv.edu (A. Dogan), TeixeiraR@uhv.edu (R. Teixeira)

Received: 09.06.2020; Accepted: 05.02.2021
} 
As the reality is generally more cruel than the old story, Park and Teixeira in [6] introduced a more complicated and cruel version of the problem in which one cannot easily figure out how to survive in the same situation.

First, we shall introduce basic definitions and notations. Throughout this paper $n$ will denote the number of people and they will be labeled from 0 to $n-1$ around the circle. $s$ will denote the number of people who are skipped and $k$ will denote the number of people who are executed in each iteration. Here the numbers $n, k$, and $s$ are positive integers and $n \geq k$ unless otherwise stated.

We start with the person 0 on a circle and apply the following iteration. We skip $s$ people and then execute $k$ people proceeding around the circle. The iteration is repeated until there are at most $k$ people left. The case where there is no survivor is omitted. Following the notation introduced in [6], we shall denote the total number of iterations and survivors by $I$ and $P$, respectively, and they are given by the formulas below.

Proposition $1.1([6])$.

and

$$
I=\left\{\begin{array}{lll}
\left\lfloor\frac{n}{k}\right\rfloor & \text { if } n \not \equiv 0 & (\bmod k) \\
\left\lfloor\frac{n}{k}\right\rfloor-1 & \text { if } n \equiv 0 & (\bmod k)
\end{array}\right.
$$

$$
P=\left\{\begin{array}{lll}
n \bmod k & \text { if } n \neq 0 & (\bmod k) \\
k & \text { if } n \equiv 0 & (\bmod k) .
\end{array}\right.
$$

The Generalized Josephus Problem, which is denoted by $J(n, s, k ; i)$, is to determine the initial position of the $i_{t h}$ survivor after $I$ iterations. With this notation, the original Josephus Problem is equivalent to $J(n, 1,1 ; 1)$.

Now we will give an example that will make easier for readers to follow the notation introduced.

Example 1.2. Consider $J(12,2,3 ; 2)$. We label a number to each person starting from 0 to 11 , and starting from the first person, we skip two people $(s=2)$ followed by executing three people $(k=3)$. Then, we repeat the iteration until there are at most 3 people. Since $12 \equiv 0(\bmod 3)$ and $\left\lfloor\frac{12}{3}\right\rfloor-1=3$, the total number of iterations is $I=3$ and the number of survivors at the end is $P=3$. The following is the initial order of people on the circle:

$$
\text { (0)(1) } 2 \text { (3)(4)(5) } 6 \text { (7) } 8 \text { (9)(10)(11) }
$$

Note that each iteration consists of two parts: skipping and executing. First, we skip 2 people which is equivalent to rotating the circle by two positions. Then we have

$$
\text { (2)(3)(4)(5)(6)(7) 8)(9)(10)(11)(1) (1) }
$$

Now we execute 3 people and it gives

$$
\text { (5) (6) (7) } 8 \text { (9)(10)(11)(10) }
$$

This is the result of the first iteration. Note that the starting person is now (5). We apply another iteration; namely we skip 2 people and then execute 3 people, which yields

$$
\text { (10)(11)(1) (1) } 5 \text { (6) }
$$

Now the starting person is (10). After applying another iteration, we have

$$
\text { (6) (11) }
$$

Now we have three people which is less than or equal to the number of executions, so we stop. This implies that we have three survivors. The first survivor is (6), the second (10), and the third (11). Thus, the initial position of the second survivor is 10 , that is, the $11_{t h}$ person in the original circle will be the second survivor. 
One can see from this example that the position of the $i_{t h}$ survivor at the end is $i-1$. Note that the positions of people starting from 0 are consistent with modular operation. To find explicit formulas, we will take a backward approach which is similar to the one in [6]. In the above example, we can see that the position of (10), the second survivor, at the end is 1 . Her or his position before the last iteration is 0 . The position before that is 5 , and the beginning position is 10 . By tracing the positions of survivors relative to the starting person in each iteration back to the beginning, we could find the initial position of the survivors.

We shall now state definitions and notations following [6].

Definition $1.3([6])$. For $0 \leq j \leq I, S_{i}(j)$ denotes the position of the $i_{t h}$ survivor and $N(j)$ represents the number of people at the $(I-j)_{t h}$ iteration.

Notice that $S_{i}(I)$ represents the initial position of the $i_{t h}$ survivor, $S_{i}(0)$ is the position of the $i_{t h}$ survivor at the end, and the number of people at the end is $N(0)=P$. Also, for $1 \leq i \leq P, S_{i}(0)=i-1$.

Moreover, one can write the recurrence relation: for $1 \leq i \leq P$ and $0 \leq j \leq I-1$,

$$
\begin{aligned}
& N(j+1)=N(j)+k \\
& S_{i}(j+1)=S_{i}(j)+(k+s) \bmod N(j+1)
\end{aligned}
$$

Definition $1.4([6])$. The $m_{t h}$ crossover of the $i_{t h}$ survivor is the iteration number $j$ such that $0 \leq S_{i}(j)<s$ for the $m_{t h}$ time, and is denoted by $C r_{i}(m)$. Without loss of generality, we define $C r_{i}(0)=0$.

In Example 1.2, the first crossover of (6) is 2, that is, $C r_{1}(1)=2$ and $C r_{2}(1)=C r_{3}(1)=1$. Since the final positions of the survivors are different, so are the behaviors of $C r_{i}(\cdot)$ for $1 \leq i \leq P$.

Now we state and prove a useful result that shall be used later in the paper.

Proposition 1.5. For any nonnegative integer $m$,

$$
C r_{i}(m+1)=C r_{i}(m)+\left\lceil\frac{N\left(C r_{i}(m)\right)-S_{i}\left(C r_{i}(m)\right)}{s}\right\rceil .
$$

Proof. At the $m_{t h}$ crossover, by the definition, the number of people is $N\left(C r_{i}(m)\right)$ and the position of the $i_{t h}$ survivor is $S_{i}\left(C r_{i}(m)\right)$. Note that $N(\cdot)$ increases by $k$ and $S_{i}(\cdot)$ increases by $s+k$ at each iteration. Thus the number of iterations required for the next crossover is the smallest integer $l$ satisfying

$$
N\left(C r_{i}(m)\right)+l k \leq S_{i}\left(C r_{i}(m)\right)+l(s+k) .
$$

That is;

$$
\frac{N\left(C r_{i}(m)\right)-S_{i}\left(C r_{i}(m)\right)}{s} \leq l .
$$

Since $l$ is an integer, we must have

$$
l=\left\lceil\frac{N\left(C r_{i}(m)\right)-S_{i}\left(C r_{i}(m)\right)}{s}\right\rceil .
$$

Hence, we have

$$
C r_{i}(m+1)=C r_{i}(m)+l=C r_{i}(m)+\left\lceil\frac{N\left(C r_{i}(m)\right)-S_{i}\left(C r_{i}(m)\right)}{s}\right\rceil
$$

as desired.

The serial execution Josephus Problem was the main topic of [6], and we present its main result below. 
Theorem 1.6. In $J(n, 1, k ; i)$ with $P>0$ survivors, for $1 \leq i \leq P$, suppose $n$ can be decomposed as

$$
n=(P+(P-i+1) k) \cdot(k+1)^{\alpha}+\beta \cdot k
$$

where $\alpha$ and $\beta$ are nonnegative integers and $\alpha$ is the highest such power. Then the initial position of the $i_{\text {th }}$ survivor $S_{i}(I)$ is given by

$$
S_{i}(I)=\beta \cdot(k+1) .
$$

Now the original Josephus Problem is a direct consequence of Theorem 1.6 with $k=1$, $P=1$, and $i=1$.

Corollary 1.7 (Folklore). In $J(n, 1,1 ; 1)$, let $n=2^{\alpha}+\beta$, where $\alpha$ and $\beta$ are nonnegative integers and $\alpha$ is the highest such power. Then the initial position of the survivor is $S_{1}(I)=2 \beta$.

\section{Block Josephus Problem $J(n, k, k ; i)$}

In this section, we will focus on the Block Josephus Problem, $J(n, k, k ; i)$ where the number of skips and the number of executions are equal. Since the original Josephus Problem has been studied thoroughly, we assume that $k \geq 2$ here. The problem shall be divided into the following four cases and each case is studied separately.

(1) when there are $k$ survivors, that is; $P=k$

(2) when there is exactly one survivor, that is; $P=1$

(3) when there are $k-1$ survivors, that is; $P=k-1$

(4) when the number of survivors is between 1 and $k-1$, that is; $1<P<k-1$

We will state and prove nonrecursive formulas for the first three cases. We will also include a discussion for the case when $1<P<k-1$ in the last section of the paper.

\section{1. $J(n, k, k ; i)$ with $P=k$}

In $J(n, k, k ; i)$, suppose that the number of survivors is $P=k$. Then $n \equiv 0(\bmod k)$. So, we can divide $n$ people into $n / k$ groups. Thus, at each iteration, skipping $k$ people is the same as skipping one group of $k$ people and executing $k$ people is equivalent to executing one group of $k$ people. This observation implies that $J(n, k, k ; i)$ is equivalent to $J\left(\frac{n}{k}, 1,1 ; 1\right)$, the original Josephus Problem.

\section{2. $J(n, k, k ; 1)$ with $P=1$}

Now we study the case with only one survivor. It is crucial to understand the behavior of $C r_{i}(\cdot)$ to solve the problem. So, we start with an example.

Example 2.1. Consider $J(n, 4,4 ; 1)$. Table 1 shows the number of people $N\left(C r_{1}(m)\right)$ and the position of the survivor $S_{1}\left(C r_{1}(m)\right)$ at the $m_{t h}$ crossover for $m \geq 0$.

From this example, we notice the following patterns in $N\left(C r_{1}(\cdot)\right)$ and $S_{1}\left(C r_{1}(\cdot)\right)$ :

\section{Observation 2.2.}

$$
N\left(C r_{1}(m+1)\right)= \begin{cases}2 N\left(C r_{1}(m)\right)+k-1 & \text { if } S_{1}\left(C r_{1}(m)\right)=0 \\ 2 N\left(C r_{1}(m)\right)-1 & \text { if } S_{1}\left(C r_{1}(m)\right) \neq 0\end{cases}
$$

and

$$
S_{1}\left(C r_{1}(m+1)\right)=S_{1}\left(C r_{1}(m)\right)-1 \bmod k
$$

Based on this observation, we can build formulas for $N\left(C r_{1}(m)\right)$ and $S_{1}\left(C r_{1}(m)\right)$ for any positive integer $m$. 


\begin{tabular}{|c|c|c|}
\hline$m$ & $S_{1}\left(C r_{1}(m)\right)$ & $N\left(C r_{1}(m)\right)$ \\
\hline 0 & 0 & 1 \\
1 & 3 & 5 \\
2 & 2 & 9 \\
3 & 1 & 17 \\
4 & 0 & 33 \\
5 & 3 & 69 \\
6 & 2 & 137 \\
7 & 1 & 273 \\
8 & 0 & 545 \\
9 & 3 & 1093 \\
$\vdots$ & $\vdots$ & $\vdots$ \\
\hline
\end{tabular}

Table 1. $J(n, 4,4 ; i)$ with 1 survivor

Theorem 2.3. For any positive integer $m$, let $\beta=m \bmod k$. Then

$$
N\left(C r_{1}(m)\right)= \begin{cases}\frac{k}{2}\left(\frac{2^{m+k}-2^{k}}{2^{k}-1}\right)+1 & \text { if } \beta=0 \\ \frac{k}{2}\left(\frac{2^{m+k}-2^{\beta}}{2^{k}-1}\right)+1 & \text { if } \beta \neq 0\end{cases}
$$

and

$$
S_{1}\left(C r_{1}(m)\right)=k-\beta .
$$

Before we prove Theorem 2.3, we will investigate the number of people after $C r_{1}(1)$ iterations, that is after the first crossover.

Lemma 2.4. $N\left(C r_{1}(1)\right)=1+k$ and $S_{1}\left(C r_{1}(1)\right)=k-1$.

Proof. Since $S_{1}(0)=0$ and $N(0)=1$, by Proposition 1.5, we have

$$
C r_{1}(1)=\left\lceil\frac{N(0)-S_{1}(0)}{k}\right\rceil=\left\lceil\frac{1}{k}\right\rceil=1 .
$$

Thus

$$
N\left(C r_{1}(1)\right)=1+C r_{1}(1) \cdot k=1+k
$$

and also we have

$$
\begin{aligned}
S_{1}\left(C r_{1}(1)\right) & =S_{1}(0)+2 k l \bmod N\left(C r_{1}(1)\right) \\
& =0+2 k \bmod (1+k) \\
& =k-1 .
\end{aligned}
$$

Proof of Theorem 2.3. We will prove this using the mathematical induction on the crossing number $m$. For the base case, let $m=1$. Then $\beta=1$ as $1 \bmod k=1$. So, we have

and

$$
N\left(C r_{1}(1)\right)=\frac{k}{2}\left(\frac{2^{1+k}-2}{2^{k}-1}\right)+1=k+1
$$

$$
S_{1}\left(C r_{1}(1)\right)=k-1
$$

which are consistent with Lemma 2.4. This proves the base case.

Now assume that the claim is true for any positive integer $m>1$. We split the proof into the following three cases depending on the values of $\beta$, and we shall consider each case separately. 
- $\beta=k-1$, that is, $m \equiv k-1(\bmod k)$,

- $\beta=0$, that is, $m \equiv 0(\bmod k)$, and

- $1 \leq \beta<k-1$, that is $1 \leq m \bmod k<k-1$.

Case 1 : Suppose that $\beta=k-1$. By Proposition 1.5, the number of iterations $l$ required from $C r_{1}(m)$ to $C r_{1}(m+1)$ is

$$
l=\left\lceil\frac{N\left(C r_{1}(m)\right)-S_{1}\left(C r_{1}(m)\right)}{k}\right\rceil .
$$

So, by the induction hypothesis,

$$
\begin{aligned}
l & =\left\lceil\frac{N\left(C r_{1}(m)\right)-S_{1}\left(C r_{1}(m)\right)}{k}\right\rceil \\
& =\left\lceil\left(\frac{k}{2}\left(\frac{2^{m+k}-2^{\beta}}{2^{k}-1}\right)+1-1\right) / k\right\rceil \\
& =\frac{1}{2}\left(\frac{2^{m+k}-2^{\beta}}{2^{k}-1}\right) .
\end{aligned}
$$

Thus, we have

$$
\begin{aligned}
N\left(C r_{1}(m+1)\right) & =N\left(C r_{1}(m)\right)+l \cdot k \\
& =\frac{k}{2}\left(\frac{2^{m+k}-2^{\beta}}{2^{k}-1}\right)+1+k \cdot \frac{1}{2}\left(\frac{2^{m+k}-2^{\beta}}{2^{k}-1}\right) \\
& =\frac{k}{2}\left(\frac{2^{m+1+k}-2^{\beta+1}}{2^{k}-1}\right)+1 .
\end{aligned}
$$

Since $\beta=k-1, \beta+1=k$. Thus

$$
N\left(C r_{1}(m+1)\right)=\frac{k}{2}\left(\frac{2^{m+1+k}-2^{k}}{2^{k}-1}\right)+1 .
$$

Now consider $S_{1}\left(C r_{1}(m+1)\right)$.

$$
\begin{aligned}
S_{1}\left(C r_{1}(m+1)\right) & =k-1+2 k l \quad \bmod N\left(C r_{1}(m+1)\right) \\
& =k-1+2 \cdot \frac{k}{2}\left(\frac{2^{m+k}-2^{\beta}}{2^{k}-1}\right) \quad \bmod N\left(C r_{1}(m+1)\right) \\
& =k-2
\end{aligned}
$$

Case 2 : Suppose that $\beta=0$. Then by the induction hypothesis,

$$
\begin{aligned}
l & =\left\lceil\frac{N\left(C r_{1}(m)\right)-S_{1}\left(C r_{1}(m)\right)}{k}\right\rceil \\
& =\left\lceil\left(\frac{k}{2}\left(\frac{2^{m+k}-2^{k}}{2^{k}-1}\right)+1-k\right) / k\right\rceil \\
& =\frac{1}{2}\left(\frac{2^{m+k}-2^{k}}{2^{k}-1}\right)+\left\lceil\frac{1-k}{k}\right\rceil \\
& =\frac{1}{2}\left(\frac{2^{m+k}-2^{k}}{2^{k}-1}\right)+1 .
\end{aligned}
$$


But then, we have

$$
\begin{aligned}
N\left(C r_{1}(m+1)\right) & =N_{1}\left(C r_{1}(m)\right)+l \cdot k \\
& =\frac{k}{2}\left(\frac{2^{m+k}-2^{k}}{2^{k}-1}\right)+1+k \cdot\left(\frac{1}{2}\left(\frac{2^{m+k}-2^{k}}{2^{k}-1}\right)+1\right) \\
& =\frac{k}{2}\left(\frac{2^{m+k}-2^{k}}{2^{k}-1}\right)+1+\frac{k}{2}\left(\frac{2^{m+k}-2^{k}}{2^{k}-1}\right)+k \\
& =\frac{k}{2}\left(\frac{2^{m+1+k}-2}{2^{k}-1}\right)+1 .
\end{aligned}
$$

Moreover,

$$
\begin{aligned}
S_{1}\left(C r_{1}(m+1)\right) & =0+2 k l \quad \bmod N\left(C r_{1}(m+1)\right) \\
& =2 k \cdot\left(\frac{1}{2}\left(\frac{2^{m+k}-2^{k}}{2^{k}-1}\right)+1\right) \quad \bmod N\left(C r_{1}(m+1)\right) \\
& =k+\frac{k}{2}\left(\frac{2^{m+1+k}-2}{2^{k}-1}\right) \quad \bmod N\left(C r_{1}(m+1)\right) \\
& =k-1 .
\end{aligned}
$$

Case 3 : Suppose that $1 \leq \beta<k-1$. Then, by the induction hypothesis,

$$
\begin{aligned}
l & =\left\lceil\frac{N\left(C r_{1}(m)\right)-S_{1}\left(C r_{1}(m)\right)}{k}\right\rceil \\
& =\left\lceil\left(\frac{k}{2}\left(\frac{2^{m+k}-2^{\beta}}{2^{k}-1}\right)+1-(k-\beta)\right) / k\right\rceil \\
& =\frac{1}{2}\left(\frac{2^{m+k}-2^{\beta}}{2^{k}-1}\right)-1+\left\lceil\frac{1+\beta}{k}\right\rceil \\
& =\frac{1}{2}\left(\frac{2^{m+k}-2^{\beta}}{2^{k}-1}\right)
\end{aligned}
$$

as $1 \leq \beta<k-1$. Now using this value of $l$, we have

$$
\begin{aligned}
N\left(C r_{1}(m+1)\right) & =N\left(C r_{1}(m)\right)+l \cdot k \\
& =\frac{k}{2}\left(\frac{2^{m+k}-2^{\beta}}{2^{k}-1}\right)+1+k \cdot \frac{1}{2}\left(\frac{2^{m+k}-2^{\beta}}{2^{k}-1}\right) \\
& =\frac{k}{2}\left(\frac{2^{m+1+k}-2^{\beta+1}}{2^{k}-1}\right)+1
\end{aligned}
$$

and also

$$
\begin{aligned}
S_{1}\left(C r_{1}(m+1)\right) & =k-\beta+2 k l \quad \bmod N\left(C r_{1}(m+1)\right) \\
& =k-\beta+2 k \cdot \frac{1}{2}\left(\frac{2^{m+k}-2^{\beta}}{2^{k}-1}\right) \quad \bmod N\left(C r_{1}(m+1)\right) \\
& =k-\beta-1
\end{aligned}
$$

which completes the proof of the theorem. 
Theorem 2.5. In $J(n, k, k ; 1)$, let $m$ be a positive integer satisfying

$$
N\left(C r_{1}(m)\right) \leq n<N\left(C r_{1}(m+1)\right) .
$$

Then

$$
n=N\left(C r_{1}(m)\right)+c \cdot k
$$

and

$$
S_{1}(I)=k-\beta+c \cdot 2 k
$$

where $\beta=m \bmod k$ and $c$ is a nonnegative integer.

Proof. For any given positive integer $n$, we can find the number of crossovers $m$ satisfying $N\left(C r_{1}(m)\right) \leq n<N\left(C r_{1}(m+1)\right)$. Then $n$ can be expresses as

$$
n=N\left(C r_{1}(m)\right)+c \cdot k
$$

where $c$ is a nonnegative integer. Notice that $c$ is the number of iterations from $C r_{1}(m)$ to $I$. Let $\beta=m \bmod k$. Then, by Theorem $2.3, S_{1}\left(C r_{1}(m)\right)=k-\beta$. So

$$
S_{1}\left(C r_{1}(m)\right)=S_{1}(I-c)=k-\beta .
$$

Thus

$$
S_{1}(I)=k-\beta+c \cdot 2 k \text {. }
$$

Example 2.6. Consider $J(1870,7,7 ; 1)$. Since $1870 \bmod 7=1$, there will be only one survivor. We want to find the initial position of the survivor. First we find $m$ such that

$$
N\left(C r_{1}(9)\right)=1807 \leq 1870<N\left(C r_{1}(10)\right)=3613 .
$$

As $m=9$ and $9 \bmod 7=2$, we have $\beta=2$. Then by Theorem 2.3 ,

$$
S_{1}\left(C r_{1}(9)\right)=S_{1}(I-c)=7-2=5 .
$$

Note that the number of iterations required from $C r_{1}(9)$ to $I$ is $(1870-1807) / 7=9$. So, by Theorem 2.5 ,

$$
S_{1}(I)=5+2 \cdot 7 \cdot 9=131 .
$$

Hence the $132_{n d}$ person will be the survivor.

\section{3. $J(n, k, k ; i)$ with $P=k-1$}

In this section, we will investigate $J(n, k, k ; i)$ with $P=k-1$, that is, when the number of survivors at the end is $k-1$. Before we state and prove our main theorem for this section, in order to have an intuition of the problem, we shall consider an example $J(n, 4,4 ; i)$ with 3 survivors.

Example 2.7. Consider $J(n, 4,4 ; i)$ with 3 survivors. Table 2 shows $N\left(C r_{i}(m)\right)$, the number of people, and $S_{1}\left(C r_{1}(m)\right)$, the position of the $i_{t h}$ survivor, at the $m_{t h}$ crossover for $m \geq 0$. 


\begin{tabular}{|c|c|c|c|c|c|c|}
\hline$m$ & $S_{1}(\cdot)$ & $N(\cdot)$ & $S_{2}(\cdot)$ & $N(\cdot)$ & $S_{3}(\cdot)$ & $N(\cdot)$ \\
\hline 0 & 0 & 3 & 1 & 3 & 2 & 3 \\
1 & 1 & 7 & 2 & 7 & 3 & 7 \\
2 & 2 & 15 & 3 & 15 & 0 & 11 \\
3 & 3 & 31 & 0 & 27 & 1 & 23 \\
4 & 0 & 59 & 1 & 55 & 2 & 47 \\
5 & 1 & 119 & 2 & 111 & 3 & 95 \\
6 & 2 & 239 & 3 & 223 & 0 & 187 \\
7 & 3 & 479 & 0 & 443 & 1 & 375 \\
8 & 0 & 955 & 1 & 887 & 2 & 751 \\
9 & 1 & 1911 & 2 & 1775 & 3 & 1503 \\
$\vdots$ & $\vdots$ & $\vdots$ & $\vdots$ & $\vdots$ & $\vdots$ & $\vdots$ \\
\hline
\end{tabular}

Table 2. $J(n, 4,4 ; i)$ with 3 survivors

This example also suggests similar patterns compared to the patterns shown in Example 2.1. The main difference is that since there can be more survivors in this case, $S_{i}\left(C r_{i}(m)\right)$ and $N\left(C r_{i}(m)\right)$ are different for each survivor. Based on the example above, one can notice the following patterns.

\section{Observation 2.8 .}

$$
N\left(C r_{i}(m+1)\right)= \begin{cases}2 N\left(C r_{i}(m)\right)+1 & \text { if } S_{1}\left(C r_{1}(m)\right) \neq k-1 \\ 2 N\left(C r_{i}(m)\right)-P & \text { if } S_{1}\left(C r_{1}(m)\right)=k-1,\end{cases}
$$

and

$$
S_{i}\left(C r_{i}(m+1)\right)=S_{i}\left(C r_{i}(m)\right)+1 \bmod k .
$$

Based on this observation, we can have the following formulas:

Theorem 2.9. For all positive integer $m$, let $\beta=m+i-1 \bmod k$. Then

$$
N\left(C r_{i}(m)\right)=k\left(2^{m}-\frac{2^{m+i-1}-2^{\beta}}{2^{k}-1}\right)-1
$$

and

where $\beta=m+i-1 \bmod k$.

$$
S_{i}\left(C r_{i}(m)\right)=\beta
$$

Before we prove the above theorem, we study $N(\cdot)$ and $S_{i}(\cdot)$ at the first crossover.

Lemma 2.10. $N\left(C r_{i}(1)\right)=2 k-1$ and $S_{i}\left(C r_{i}(1)\right)=i$.

Proof. Let $l=C r_{i}(1)$. By Proposition 1.5, we have $l=\left\lceil\frac{N(0)-S_{i}(0)}{k}\right\rceil=\left\lceil\frac{(k-1)-(i-1)}{k}\right\rceil=1$ since $1 \leq i \leq k-1$. Using this value of $l$, we obtain $N\left(C l_{i}(1)\right)=(k-1)+k l=2 k-1$ and, thus

$$
\begin{aligned}
S_{i}\left(C l_{i}(1)\right) & =(i-1)+2 k l \quad \bmod N\left(C l_{i}(1)\right) \\
& =i-1+2 k \bmod (2 k-1)=i .
\end{aligned}
$$

Proof of Theorem 2.9. We will use induction on $m$. For $m=1$, we have $m+i-1=i$, and thus $\beta=i$. Therefore, we have

$$
\begin{aligned}
N\left(C r_{i}(1)\right) & =k\left(2^{1}-\frac{2^{1+i-1}-2^{\beta}}{2^{k}-1}\right)-1 \\
& =2 k-1,
\end{aligned}
$$


and $S_{i}\left(C r_{i}(1)\right)=\beta=i$, which are consistent with the result of Lemma 2.10.

Now for $m>0$, assume that

$$
N\left(C l_{i}(m)\right)=k\left(2^{m}-\frac{2^{m+i-1}-2^{\beta}}{2^{k}-1}\right)-1 \quad \text { and } \quad S_{i}\left(C l_{i}(m)\right)=\beta,
$$

where $\beta=m+i-1 \bmod k$. Since we have $N\left(C l_{i}(m+1)\right)=N\left(C l_{i}(m)\right)+l k$, where $l=\left\lceil\frac{N\left(C l_{i}(m)\right)-S_{i}\left(C l_{i}(m)\right)}{k}\right\rceil$, substituting the expression of $N\left(C l_{i}(m)\right)$ gives

$$
\begin{aligned}
l & =\left\lceil\frac{N\left(C l_{i}(m)\right)-S_{i}\left(C l_{i}(m)\right)}{k}\right\rceil \\
& =\left\lceil 2^{m}-\left(\frac{2^{m+i-1}-2^{\beta}}{2^{k}-1}\right)-\frac{1+\beta}{k}\right\rceil,
\end{aligned}
$$

To prove the inductive step, we consider the following two cases; when $\beta=k-1$ and when $0 \leq \beta<k-1$.

First, suppose $\beta=k-1$. As $\frac{1+\beta}{k}=\frac{1+k-1}{k}=1$, then Equation (2.1) becomes

$$
l=2^{m}-\left(\frac{2^{m+i-1}-2^{\beta}}{2^{k}-1}\right)-1 .
$$

Hence

$$
\begin{aligned}
N\left(C l_{i}(m+1)\right) & =k\left(2^{m}-\frac{2^{m+i-1}-2^{\beta}}{2^{k}-1}\right)-1+k l \\
& =k\left(2^{m}-\frac{2^{m+i-1}-2^{\beta}}{2^{k}-1}\right)-1+k\left(2^{m}-\left(\frac{2^{m+i-1}-2^{\beta}}{2^{k}-1}\right)-1\right) \\
& =k\left(2^{m+1}-\frac{2^{m+i}-2^{k}}{2^{k}-1}\right)-1-k \\
& =k\left(2^{m+1}-\frac{2^{m+i}-1}{2^{k}-1}\right)-1,
\end{aligned}
$$

and, moreover, we have

$$
\begin{aligned}
S_{i}\left(C l_{i}(m+1)\right) & =S_{i}\left(C l_{i}(m)\right)+2 k l \bmod N\left(C l_{i}(m+1)\right) \\
& =(k-1)+2 k\left(2^{m}-\frac{2^{m+i-1}-2^{\beta}}{2^{k}-1}-1\right) \bmod N\left(C l_{i}(m+1)\right) \\
& =(k-1)+k\left(2^{m+1}-\frac{2^{m+i}-2^{k}}{2^{k}-1}-2\right) \bmod N\left(C l_{i}(m+1)\right) \\
& =k\left(2^{m+1}-\frac{2^{m+i}-1}{2^{k}-1}\right)-1 \bmod N\left(C l_{i}(m+1)\right) \\
& =0 .
\end{aligned}
$$

Now suppose that $0 \leq \beta<k-1$. Since $0<\frac{1+\beta}{k}<1$, Equation (2.1) gives

$$
l=2^{m}-\frac{2^{m+i-1}-2^{\beta}}{2^{k}-1} .
$$


Thus

$$
\begin{aligned}
N\left(C l_{i}(m+1)\right) & =k\left(2^{m}-\frac{2^{m+i-1}-2^{\beta}}{2^{k}-1}\right)-1+k l \\
& =k\left(2^{m}-\frac{2^{m+i-1}-2^{\beta}}{2^{k}-1}\right)-1+k\left(2^{m}-\frac{2^{m+i-1}-2^{\beta}}{2^{k}-1}\right) \\
& =k\left(2^{m+1}-\frac{2^{m+i}-2^{\beta+1}}{2^{k}-1}\right)-1,
\end{aligned}
$$

and

$$
\begin{aligned}
S_{i}\left(C l_{i}(m+1)\right) & \left.=S_{i}\left(C l_{i}(m)\right)+2 k l \bmod N\left(C l_{i}(m+1)\right)\right) \\
& =\beta+2 k\left(2^{m}-\frac{2^{m+i-1}-2^{\beta}}{2^{k}-1}\right) \bmod N\left(C l_{i}(m+1)\right) \\
& =\beta+k\left(2^{m+1}-\frac{2^{m+i}-2^{\beta+1}}{2^{k}-1}\right) \bmod N\left(C l_{i}(m+1)\right) \\
& =\beta+1 .
\end{aligned}
$$

This completes the proof.

Theorem 2.11. In $J(n, k, k ; i)$, suppose $m$ is a positive integer such that

$$
N\left(C r_{i}(m)\right) \leq n<N\left(C r_{i}(m+1)\right) .
$$

Then $S_{i}(I)=\beta+c \cdot 2 k$, where $\beta=m+i-1 \bmod k$.

Proof. Suppose $m$ is a positive integer such that $N\left(C r_{i}(m)\right) \leq n<N\left(C r_{i}(m+1)\right)$, that is, $m$ is the largest crossover. Then the total number of iterations is $I=C r_{i}(m)+c$ where $c$ is a nonnegative integer. Since $N_{i}(\cdot)$ increases by $k$ at each iteration, $n=N\left(C r_{i}(m)\right)+c \cdot k$.

Now let $\beta=m+i-1 \bmod k$. Then, by Theorem 2.9, $S_{1}\left(C r_{1}(m)\right)=\beta$. We obtain $S_{i}\left(C r_{i}(m)\right)=S_{i}(I-c)=\beta$, and thus $S_{1}(I)=\beta+c \cdot 2 k$.

Example 2.12. Consider $J(1854,5,5 ; i)$. Since $1854 \equiv 4(\bmod 5)$, there will be 4 survivors at the end. We will identify the initial positions of the survivors and we shall start with the 1st survivor. First, we need to find $m$ such that

$$
N\left(C r_{1}(m)\right) \leq 1854<N\left(C r_{1}(m+1)\right) .
$$

By Theorem 2.4, the number of people at $m_{t h}$ crossover for the $i_{t h}$ survivor is

$$
N\left(C r_{i}(m)\right)=5 \cdot 2^{m}-1-5\left(\frac{2^{m+i-1}-2^{\beta}}{2^{5}-1}\right)
$$

where $\beta=m+i-1 \bmod k$. Therefore, we find $N\left(C r_{1}(8)\right)=1239 \leq 1854<N\left(C r_{1}(9)\right)=$ 2479 . As $\beta=8+1-1 \bmod 5=3$, the position of the $1_{s t}$ survivor at the $8_{t h}$ crossover is 3 , and also the number of iterations from $N\left(C r_{1}(8)\right)$ to $N(I)$ is $(1854-1239) / 5=123$. By Theorem 2.11, we conclude that $S_{1}\left(C r_{1}(8)\right)=S_{1}(I-123)=3$ and that $S_{1}(I)=$ $3+123 \cdot 2 \cdot 5=1233$. Thus, the first survivor is the $1234_{t h}$ person in the initial circle.

One can easily find the positions of other survivors using the same method. Namely $S_{2}(I)=1314, S_{3}(I)=1480$, and $S_{4}(I)=1811$.

\section{Conclusion and open problems}

We provided the explicit formulas for the positions of the survivors in the Block Josephus problem $J(n, k, k ; i)$ for the the cases when there is only one survivor, when there are $k-1$, and when there are $k$ survivors. The other case when there are $P$ survivors with $1<P<k-1$ is still open. We state the following observation that we believe it would help understand this case. 
Observation 3.1. $N_{i}\left(C r_{i}(m+1)\right)= \begin{cases}2 N\left(C r_{i}(m)\right)+k-P & \text { if } S_{1}\left(C r_{1}(m)\right)<P \\ 2 N\left(C r_{i}(m)\right)-P & \text { if } S_{1}\left(C r_{1}(m)\right) \geq P\end{cases}$ and $S_{i}\left(C r_{i}(m+1)\right)=S_{i}\left(C r_{i}(m)\right)-P \bmod k$.

The authors think that the difficulty in this case is that $N\left(C r_{i}(\cdot)\right)$ and $S_{i}\left(C r_{i}(\cdot)\right)$ interact each other more at each iteration. This will be a great problem for future study.

The general case of the Josephus Problem $J(n, s, k ; i)$ is still open. Especially, when $s, k>1$ and $s \neq k$, the behavior of the crossovers seems quite random. So, it will be very interesting to find different approaches to solve the problem.

It will also be quite interesting to find applications of the Josephus Problem in different areas such as graph theory and computer science. Especially, the Feline Josephus problem [8] where each person has multiple lives can be closely related to scheduling problem in computer network, operating system, and parallel computing.

The Josephus Problem can be generalized in diverse ways. For example, the number of skips can change at each iteration. A simple case of this variation was studied in [10]. Similarly, the number of executions can vary at each iteration. Another intriguing version is one with varying direction, that is, instead of making people standing in a circle, we can consider the case where people are in line and when skip or execution hits one end of the line, it reverses. Numerous variations of the Josephus Problems are possible, and some of them will be easy to understand and other will be very complicated. But all of them will pique our curiosity.

\section{References}

[1] K. Burde, Das Problem der Abzählreime und Zahlentwicklungen mit gebrochenen Basen, J. Number Theory 26 (2), 192-209, 1987.

[2] L. Halbeisen and N. Hungerbühler, The Josephus Problem, Journal de Thèorie des Nombres de Bordeaux, 9, 303-318, 1997.

[3] F. Jakóbczyk, On the Generalized Josephus Problem, Glasg. Math. J. 14, 168-173, 1973.

[4] T. F. Josephus, The Jewish War (translated by William Whiston).

[5] A.M. Odlyzko and H.S. Wilf, Functional iteration and the Josephus problem, Glasg. Math. J. 33 (2), 235-240, 1991.

[6] J.W. Park and R. Teixeira, Serial Execution Josephus Problem, Korean J. Math. 26 (1), 1-7, 2018.

[7] W.J. Robinson, The Josephus Problem, Math. Gaz. 44 (347), 47-52, 1960.

[8] F. Ruskey and A. Williams, The Feline Josephus Problem, Theory Comput. Syst. 50 (1), 20-34, 2012.

[9] A. Shams-Baragh, Formulation of the Extended Josephus Problem, in: National Computer Conference, December 2002.

[10] S. Sharma, R. Tripathi, S. Bagai, R. Saini and N. Sharma, Extension of the Josephus Problem with Varying Elimination Steps, DU Journal of Undergraduate Research and Innovation 1 (3), 211-218, 2015.

[11] R. Teixeira and J.W. Park, Mathematical Explanation and Generalization of Penn and Teller's Love Ritual Magic Trick, Journal of Magic Research 8, 21-32, 2017.

[12] N. Thèrialut, Generalizations of the Josephus Problem, Util. Math. 58, 161-173, 2000.

[13] D. Woodhouse, The Extended Josephus Problem, Rev. Mat. Hisp.-Amer. 33 (4), 207$218,1973$. 